\title{
Identification of a distinct lipidomic profile in the osteoarthritic synovial membrane by mass spectrometry imaging
}

Citation for published version (APA):

Rocha, B., Cillero-Pastor, B., Ruiz-Romero, C., Paine, M. R. L., Canete, J. D., Heeren, R. M. A., \& Blanco, F. J. (2021). Identification of a distinct lipidomic profile in the osteoarthritic synovial membrane by mass spectrometry imaging. Osteoarthritis and Cartilage, 29(5), 750-761. https://doi.org/10.1016/j.joca.2020.12.025

Document status and date:

Published: 01/05/2021

DOI:

10.1016/j.joca.2020.12.025

Document Version:

Publisher's PDF, also known as Version of record

\section{Document license:}

Taverne

Please check the document version of this publication:

- A submitted manuscript is the version of the article upon submission and before peer-review. There can be important differences between the submitted version and the official published version of record.

People interested in the research are advised to contact the author for the final version of the publication, or visit the DOI to the publisher's website.

- The final author version and the galley proof are versions of the publication after peer review.

- The final published version features the final layout of the paper including the volume, issue and page numbers.

Link to publication

\footnotetext{
General rights rights.

- You may freely distribute the URL identifying the publication in the public portal. please follow below link for the End User Agreement:

www.umlib.nl/taverne-license

Take down policy

If you believe that this document breaches copyright please contact us at:

repository@maastrichtuniversity.nl

providing details and we will investigate your claim.
}

Copyright and moral rights for the publications made accessible in the public portal are retained by the authors and/or other copyright owners and it is a condition of accessing publications that users recognise and abide by the legal requirements associated with these

- Users may download and print one copy of any publication from the public portal for the purpose of private study or research.

- You may not further distribute the material or use it for any profit-making activity or commercial gain

If the publication is distributed under the terms of Article $25 \mathrm{fa}$ of the Dutch Copyright Act, indicated by the "Taverne" license above, 


\title{
Osteoarthritis and Cartilage
}

\section{Identification of a distinct lipidomic profile in the osteoarthritic synovial membrane by mass spectrometry imaging}

\author{
B. Rocha $\dagger$, B. Cillero-Pastor $\ddagger$, C. Ruiz-Romero $\dagger \S^{* *}$, M.R.L. Paine $\ddagger$, J.D. Cañete $\|$, \\ R.M.A. Heeren $\ddagger$, F.J. Blanco $\dagger \mathbb{q}^{*}$
}

$\dagger$ Grupo de Unidad de Proteómica, Grupo de Investigación de Reumatología (GIR), Instituto de Investigación Biomédica de A Coruña (INIBIC), Complexo Hospitalario Universitario de A Coruña (CHUAC), Sergas, Universidade da Coruña (UDC), C/ As Xubias de Arriba 84, 15006, A Coruña, Spain

$\ddagger$ The Maastricht Multimodal Molecular Imaging Institute (M4I), Division of Imaging Mass Spectrometry, Maastricht University, the Netherlands

$\S$ Centro de Investigación Biomédica en Red de Bioingeniería, Biomateriales y Nanomedicina (CIBER-BBN), Av. Monforte de Lemos, 3-5. Pabellón 11, 28029,

Madrid, Spain

I Unidad de Artritis. Servicio de Reumatología. Hospital Clínico de Barcelona, Barcelona, Spain

ฯ Universidade da Coruña (UDC), Grupo de Investigación de Reumatología y Salud (GIR-S), Departamento de Fisioterapia, Medicina y Ciencias Biomédicas,

Facultad de Fisioterapia, Campus de Oza, 15008, A Coruña, Spain

\section{A R T I C L E I N F O}

\section{Article history:}

Received 13 July 2020

Accepted 16 December 2020

\section{Keywords:}

Mass spectrometry imaging

Osteoarthritis

Synovial membrane

Glycerophospholipids

\begin{abstract}
S U M M A R Y
Objective: Synovial inflammation is one of the most characteristic events in different types of arthritis, including Osteoarthritis (OA). Emerging evidence also suggests the involvement of lipids in the regulation of inflammatory processes. The aim of this study was to elucidate the heterogeneity and spatial distribution of lipids in the OA synovial membrane and explore their putative involvement in inflammation.

Method: The abundance and distribution of lipids were examined in human synovial membranes. To this end, histological cuts from this tissue were analysed by matrix-assisted laser desorption ionization mass spectrometry imaging (MALDI-MSI). The lipidomic profile of OA synovium was characterized and compared with healthy and other forms of inflammatory arthropathies as Rheumatoid Arthritis (RA) and Psoriatic Arthritis (PsA) using principal component analysis and discriminant analysis methods. Lipid identification was undertaken by tandem MS analyses and database queries.

Results: Our results reveal differential and characteristic lipidomic profiles between OA and control samples. Specifically, we unveiled that OA synovium presents elevated levels of phosphatidylcholines, fatty acids and lysophosphatidic acids and lower levels of lysophosphatidylcholines compared to control tissues. The spatial distribution of particular glycerophospholipids was also correlated with hypertrophic, inflamed or vascularized synovial areas. Compared with other inflammatory arthritis, the OA tissue showed lower amounts of phosphatidylethanolamine-based plasmalogens.

Conclusions: This study provides a novel insight into the lipid profiles of synovial membrane and differences in abundance between $\mathrm{OA}$ and control tissues. The lipidomic alterations improves understanding of the pathogenic mechanisms of $\mathrm{OA}$ and may be important for its diagnosis.
\end{abstract}

() 2021 Osteoarthritis Research Society International. Published by Elsevier Ltd. All rights reserved.

\footnotetext{
* Address correspondence and reprint requests to: F.J. Blanco, Unidad de Proteómica, Grupo de Investigación de Reumatología (GIR), Instituto de Investigación Biomédica de A Coruña (INIBIC), Complexo Hospitalario Universitario de A Coruña (CHUAC), Sergas, Universidade da Coruña (UDC), C/ As Xubias de Arriba 84, 15006, A Coruña, Spain. Tel.: 34-981-176399; fax: 34-981-176398

** Address correspondence and reprint requests to: C. Ruiz-Romero, Unidad de Proteómica, Grupo de Investigación de Reumatología (GIR), Instituto de Investigación Biomédica de A Coruña (INIBIC), Complexo Hospitalario Universitario de A Coruña (CHUAC), Sergas, Universidade da Coruña (UDC), C/ As Xubias de Arriba 84, 15006, A Coruña, Spain.

E-mail addresses: cristina.ruiz.romero@sergas.es (C. Ruiz-Romero), fblagar@ sergas.es (F.J. Blanco).
}

\section{Introduction}

Osteoarthritis (OA) is one of the most common types of chronic arthritis. It is frequently associated with signs and symptoms of inflammation, including joint pain, swelling and stiffness, which lead to significant functional impairment and disability ${ }^{1}$. Nowadays, $\mathrm{OA}$ is considered as a whole joint disease that affects several anatomical structures in the joint, including synovial membrane inflammation ${ }^{2}$. Synovial membrane or synovium is a connective tissue that separates the articular capsule from the joint cavity. 
Under normal conditions, it is organized into two distinct tissue layers: the synovial lining (or intima) composed of 2-3 layers of type B synoviocytes or fibroblast-like synoviocytes (FLS), and the synovial sublining (or subintima). The sublining layer consists of multiple types of connective tissue: fibrous, adipose or aerolar; blood and lymphatic vessels and a low content of immune cells ${ }^{3}$.

The synovial inflammation or synovitis observed in OA patients encompasses severe histological changes. These involve the proliferation and hyperplasia of the lining layer (LL) cells, fibrosis and increased vascularity in the sublining layer, followed by a massive infiltration of immune cells ${ }^{4}$. Synovitis can be caused by the release of cartilage breakdown products into the synovial cavity that activate synovial lining cells. This activation induces the production of proinflammatory mediators, which in turn attract immune cells, increase angiogenesis and induce a phenotypic shift in chondrocytes ${ }^{5}$. Synovial damage and inflammation has been observed in all OA stages $^{6}$ and correlates with the radiographic progression of knee $\mathrm{OA}^{7}$. Therefore, insights into the cause and features of synovial pathogenesis are important to improve the understanding of OA.

Increasing evidence suggests that cytokines and different lipids are involved in regulating synovial inflammation at the molecular $\operatorname{level}^{8}$. It has been described that cytokines, chemokines and eicosanoids derived from arachidonic acid (AA), such as prostaglandins, are released during the early phases of inflammation, inducing the recruitment and activation of immune cells ${ }^{9}$. Other lipids, including fatty acids (FA), phospholipids and other bioactive lipid mediators derived from them could also contribute to OA, as reviewed recently ${ }^{10}$. For instance, alterations of the composition and concentration of glycerophospholipids and sphingolipids in the synovial fluid (SF) from OA patients have been linked to disease severity $^{11-13}$.

The most common analytical techniques employed for the largescale investigation of tissue lipids (lipidomes) include mass spectrometry (MS) combined to liquid chromatography (LC) or gas chromatography (GC) and nuclear magnetic resonance (NMR) spectroscopy ${ }^{14}$. However, these techniques do not enable a direct correlation between differentially expressed lipid profiles and tissue histology. To overcome this limitation, matrix-assisted laser desorption/ionization MS imaging (MALDI-MSI) has been recently introduced in the rheumatology field ${ }^{15}$. It is a powerful technology that allows the direct high-throughput analysis and determination of the spatial distribution of molecules on tissue sections in a single imaging experiment ${ }^{16}$. Matrix-assisted laser desorption ionization mass spectrometry imaging (MALDI-MSI) measures mass spectra at defined spatial points across the tissue surface. By these means, each point or pixel is linked to a mass spectrum containing signals from the molecules at a specific mass-to-charge $(\mathrm{m} / \mathrm{z}$ ) value that are desorbed from that tissue region. A plot of the intensity of a signal produces a map of the relative abundance of that molecule over the imaged tissue ${ }^{17}$. Therefore, MALDI-MSI is not a quantitative method, but it can be used for semi-quantitative comparisons between sample groups based on the relative abundance of the molecules.

As synovial tissue is one of the principal targets in OA, research on this tissue can improve our understanding of OA and identify potential candidates useful for disease diagnosis and stratification, as well as for predicting disease course and treatment response ${ }^{18}$. In this work, MALDI-MSI has been applied to explore alterations on the composition and distribution of lipids in human normal and OA-affected synovium. The spatial distribution of lipid species has also been correlated with histological features of $\mathrm{OA}$ synovitis. Furthermore, the characterized OA lipidomic signature has been compared with Psoriatic Arthritis (PsA) and Rheumatoid Arthritis (RA) profiles, in order to unravel the OA molecular tissue heterogeneity compared to PsA and RA.

\section{Method}

\section{Synovial procurement and processing}

Human knee synovial samples were obtained from adult donors undergoing joint surgery affected by OA $(n=13)$, and via arthroscopic biopsies from RA $(n=6)$ and PsA $(n=12)$ patients. Patients undergoing arthroscopy by traumatic event and without any type of secondary arthritis to any rheumatic disease or inflammatory joint effusion were also included in this study (control donors, $n=10$ ). Patients were diagnosed following the classification criteria determined by the American College of Rheumatology for $\mathrm{OA}^{19}$ and $\mathrm{RA}^{20}$, and according to the CASPAR criteria for PsA ${ }^{21}$. Patients with history of malignancy, underlying severe conditions or diagnosed with other joint diseases were excluded from the study. All tissue samples were provided by the Tissue Bank and the Autopsy Service at Hospital Universitario de A Coruña, Spain. Informed consent was obtained from all participants before surgery. The study was approved by the local Ethics Committee (Galicia, Spain). Demographic characteristics of the patients are summarized in Table I.

\section{Sample preparation for MALDI-MSI}

Synovial membrane tissues were removed from the knee, immediately snap frozen in liquid nitrogen and then stored at $-80^{\circ} \mathrm{C}$ until processing. Ten $\mu \mathrm{m}$ tissue sections were cut using a cryostat (Leica Microsystems, UK), mounted onto indium tin oxide (ITO)-coated glass slides (4-8 $\Omega$ resistance, Delta Technologies, Stillwater, MN, USA) and stored at $-80^{\circ} \mathrm{C}$. Tissue sections were analyzed in duplicate for each tissue and type of imaging experiment (lipids in positive mode, lipids in negative mode and metabolites). Therefore, six tissue sections were analyzed per patient. Prior to matrix application, slides were placed in a vacuum dessicator at room temperature and defrosted for $20 \mathrm{~min}$. Tissue sections were then scanned using a Nikon Super CoolScan 5000 ED (Nikon Corporation, Tokyo, Japan). Finally, matrix solutions were deposited as detailed in the Supplementary Methods.

\section{MALDI-MSI analysis of human synovium}

Lipid measurements were performed on a Bruker rapifleX MALDI Tissuetyper ${ }^{\mathrm{TM}}$ system (Bruker Daltonik GmbH, Bremen, Germany), operated in reflectron mode in both positive and negative ionization modes in the $m / z$ of $200-2000$ Da. Additionally, metabolites in OA and control tissue slides were analysed using a Bruker 9.4 T solariX FT-ICR mass spectrometer (Bruker Daltonik $\mathrm{GmbH}$, Bremen, Germany) equipped with a SmartBeam II Nd:YAG laser operated with the MALDI source. Precise procedures followed for this analysis are detailed in the Supplementary Methods. Images of the whole tissue sections were acquired using a $50 \times 50 \mu \mathrm{m}$ raster $(40 \times 40 \mu \mathrm{m}$ beam scan area). Thus, each pixel (spectrum) represents the averaged lipid profile acquired from adjacent cells within a $50 \mu \mathrm{m}$ area. MALDI-MSI images were generated using FlexImaging 4.1 software (Bruker Daltonik $\mathrm{GmbH}$ ) and normalized to compare ion distributions from different tissues. After acquisition, the tissue sections were stained in order to spatially correlate the lipid distributions with histological features.

\section{Data analysis and image generation}

Statistical analyses were performed following in-house developed procedures ${ }^{22}$ and as detailed in Supplementary Methods. After characterization of the synovium lipidome, signal intensities of lipid species were compared between control and OA synovium sections using GraphPad Prism software 7.0. Then, lipid intensities 


\begin{tabular}{|c|c|c|c|c|}
\hline & PsA $(n=12)$ & $\mathrm{RA}(n=6)$ & $\mathrm{OA}(n=13)$ & Control $(n=10)$ \\
\hline Age, years $($ mean $\pm S D)$ & $52 \pm 13$ & $67 \pm 17$ & $73 \pm 11$ & $69 \pm 9$ \\
\hline Sex (female/male) & $6 / 6$ & $4 / 2$ & $11 / 2$ & $1 / 9$ \\
\hline BMI, $\mathrm{kg} / \mathrm{m}^{2}($ mean $\pm \mathrm{SD})$ & $33 \pm 5$ & $30 \pm 0$ & $33 \pm 7$ & $25 \pm 5$ \\
\hline Total cholesterol, $\mathrm{mg} / \mathrm{dl}($ mean $\pm \mathrm{SD})$ & $201 \pm 29$ & $174 \pm 30$ & $214 \pm 64$ & $152 \pm 34$ \\
\hline Triglycerides, mg/dl (mean \pm SD) & $174 \pm 27$ & $88 \pm 27$ & $96 \pm 24$ & $105 \pm 24$ \\
\hline Glucose, mg/dl (mean \pm SD) & $96 \pm 21$ & $84 \pm 6$ & $93 \pm 27$ & $99 \pm 16$ \\
\hline $\mathrm{CRP}, \mathrm{mg} / \mathrm{dl}($ mean $\pm \mathrm{SD})$ & $1.2 \pm 1.8$ & $0.5 \pm 0.2$ & $1.4 \pm 2.9$ & $1.9 \pm 1.9$ \\
\hline
\end{tabular}

PsA, Psoriatic Arthritis; RA, Rheumatoid Arthritis; BMI, body mass index; ESR, erythrocyte sedimentation rate; CRP, C-Reactive Protein; SD, standard deviation. study

were also compared between OA vs PsA and OA vs RA. MALDI-MSI images were generated for those statistically significant altered lipids using FlexImaging 4.1 software to investigate their spatial distribution within tissues, with a $m / z$ bin width of $\Delta m / z=0.05$ Da. Regions of interest based on the histological changes associated to OA were annotated. Then, lipid intensities extracted from each synovial compartment (LL, sublining layer (SL) and blood vessels (BV)) were compared.

Pathway analysis was carried out using the list of significantly different lipids between control and OA synovial tissues using MetaboAnalyst 4.0. and KEGG (Kyoto Encyclopedia of Genes and Genomes) as metabolic pathway library. Pathway enrichment was determined by Hypergeometric test and a False Discovery Rate (FDR)-adjusted $P$-value of 0.05 was used as cut-off value.

\section{MALDI-MS/MS for identification}

$M / z$ values with the highest scale loading in the first discriminant function (DF1) were targeted directly from tissue sections for MS fragmentation and database searching in order to confirm the molecular identity of the corresponding lipids and metabolites (see Supplementary Methods for details). As lipid identification is restricted by the sensitivity of the technology, the minor components of the lipid ion species were not entirely characterized directly from the tissue using MS/MS analysis due to their low abundance.

\section{Statistics}

Statistical analyses were carried out using the SPSS program. A $P$-value $<0.05$ was considered statistically significant and statistical tests were two-sided. Two-group comparisons of the lipid intensities (abundances) were carried out with the Mann-Whitney $U$ test using GraphPad Prism software 7.0. Data are presented in the figure legends or text as mean intensity $\pm 95 \%$ confidence interval.

\section{Results}

MALDI-MSI analysis reveals a characteristic lipidomic profile in the OA synovium

MALDI-MSI was used to study the profile and distribution of lipids in control and OA synovial membranes. After imaging experiments, data from control and OA groups were analysed by multivariate statistical methods. Principal component analysis
(PCA) in combination with DA showed a good separation between control and OA synovial membranes based on their lipid profiles [Fig. 1(A)-(C)]. Several specific lipid masses of each group obtained by MALDI-MSI are also represented in Fig. 1(B)-(D). A total of 62 lipid species were identified and grouped into three major lipid classes: FA, sphingolipids, and glycerophospholipids (GP), which represent $9 \%, 5 \%$ and $87 \%$ of the total of annotated lipid species, respectively (Supplementary Fig. 1). Of these identified lipid species, 52 were significantly different between OA and control synovial membranes ( $P$-value $<0.05)$ (Supplementary Table 1 ). Among the altered lipids, GP were significantly increased in OA compared to control tissues, including phosphatidylcholines (PC), phosphatidylethanolamines (PE), phosphatidylserines (PS) and phosphatidylinositols (PI). Normalized intensity and spatial distribution of six different PC between OA and control synovial membranes are shown in Supplementary Fig. 2. More representative examples of the lipids differentially abundant between study groups are presented in Supplementary Fig. 3. Several FA, such as AA, and lysophosphatidic acids (LPA) also showed a significantly higher abundance in OA samples related to controls (Fig. 2). In contrast, lysophosphatidylcholines (LPC) were decreased in OA.

In order to elucidate potential metabolic pathways that were differentially regulated between control and OA sample groups, the list of 52 significantly modulated lipids was uploaded into MetaboAnalyst for Pathway Analysis. The original and adjusted $P$-values together with impact factors are represented in Supplementary Fig. 4(A) and (B). In this analysis, we identified GP metabolism as significant metabolic pathway in OA vs control with an impact factor of 0.37 and $P$-value $\leq 0.05$ after FDR correction.

\section{Correlation between spatial distribution of lipids and OA-associated synovial features}

In addition to the differences between control and OA synovium regarding their lipidomic profiles, we also observed distinctive intensity differences of some PC between the lining and sublining layers in the diseased tissue. PCA of individual OA synovial membranes separated the sublining from the intimal layer, based on the higher content of $\mathrm{PC}$ in the latter [Fig. 3(A) and (B)]. Additionally, HCA showed that several PC, such as PC (16:0_18:1), PC (18:1_18:2), PC (18:0_18:1) and PC (18:1_18:1) were grouped in the same cluster, indicating a similar spatial distribution and co-localization in the synovial lining layer (3C-D).

Moreover, the co-registration of the MALDI-MSI images with the optical images of the stained tissue sections allowed to correlate 
A

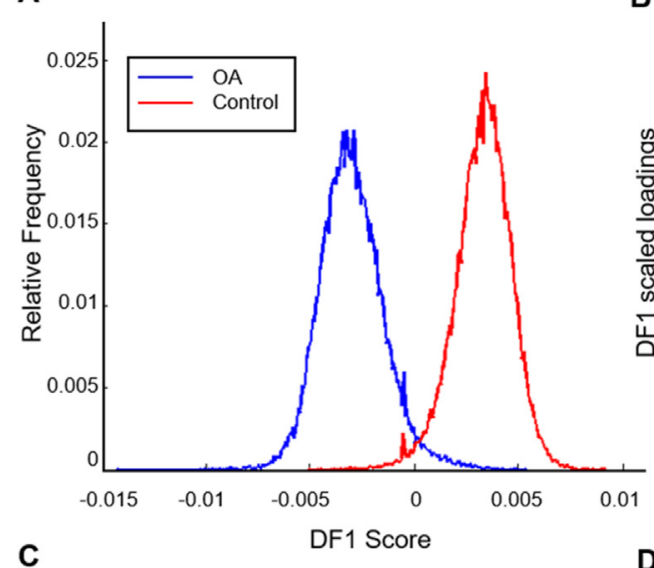

。

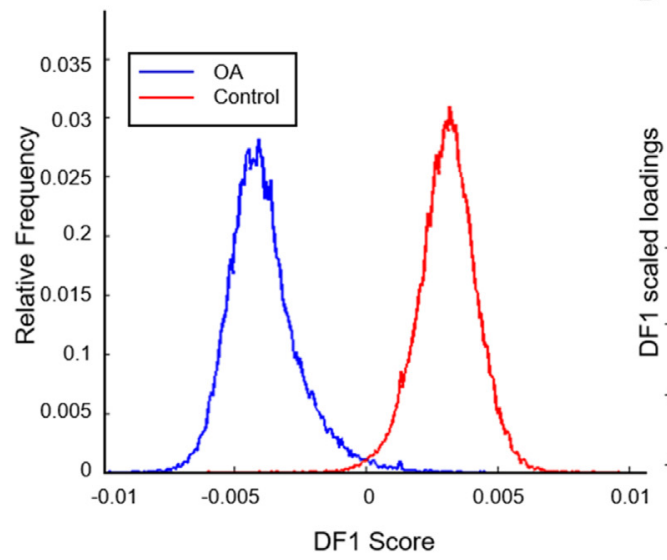

B

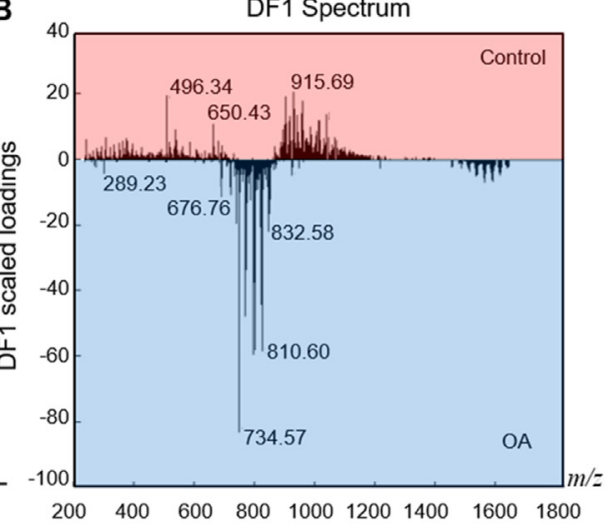

D

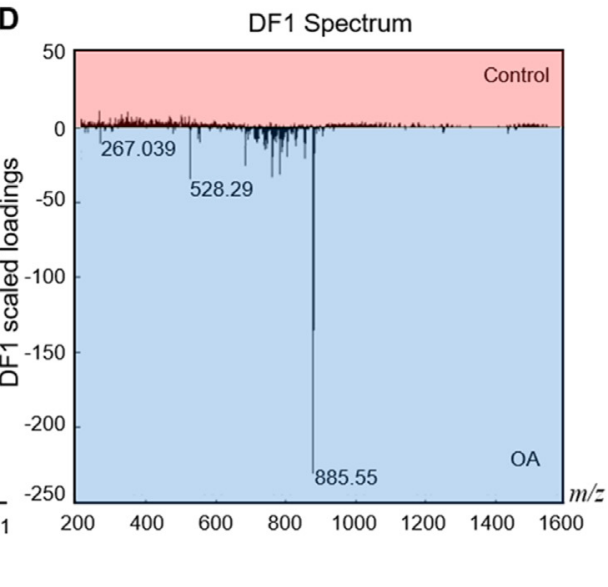

\section{Fig. 1}

Principal component analysis (PCA) and discriminant analysis (DA) of control and OA-affected synovial membranes. A-C) Histogram score plots of the PCA-DA analysis discriminating OA and control samples based on lipid profiles identified in positive $(A)$ and negative $(C)$ ionization mode, respectively. B-D) DF1 spectra loading plots showing the discriminating lipid masses between $O A$ and control synovial DF1 spectra loading plots showing the discriminating lipid masses
membranes identified in positive (B) and negative (D) ionization mode. 


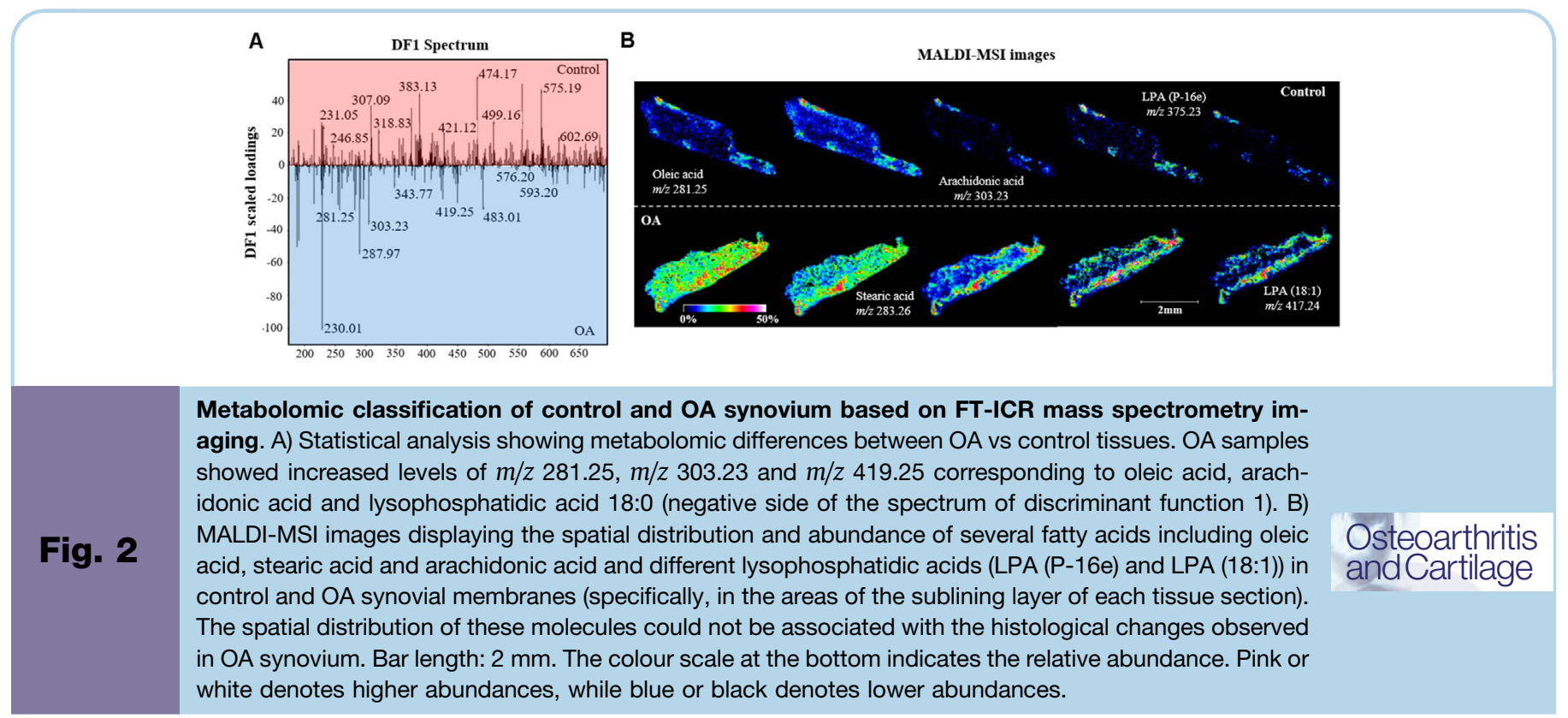

the spatial distribution of PC (18:1_18:1) with the histological features of synovial inflammation. PC (18:1_18:1) was predominantly observed in the hypertrophic LL of the OA synovial membrane [Fig. 4(A)]. Regarding other spatial lipid changes, $m / z 810.53$ corresponding to PS (18:0_20:4) was enriched in areas characterized by BV and diffuse inflammatory cell aggregates observed in the OA subintima layer but not in control tissues [Fig. 4(B)]. Although these changes could potentially be affected by lipids derived from local BV via diffusion, we observed that this effect was restricted to the $\mathrm{BV}$ areas, since the heme group showed little to no signal in the tissue surrounding those regions.

To confirm the visual differences regarding the tissue location of PC (18:1_18:1) and PS (18:0_20:4), we compared the normalized signal intensities extracted from each synovial compartment, including LL, sublining layer (SL) and BV, from those biopsies with a preserved morphology [Fig. 4(C) and (D)]. Interestingly, PS (18:0_20:4) co-localized with other PS and DMPE species according to clustering analysis (Supplementary Fig. 5). The preferential location of PC (18:1_18:1) and PS (18:0_20:4) within OA synovial membranes, as well as other GP that presented characteristic spatial distributions, are reported in Supplementary Table 2.

\section{Distinctive lipidomic signature of the OA synovium compared to} other inflammatory arthritis

PCA-DA analyses were then performed to evaluate the lipidomic disturbances associated specifically with OA. To this aim, the lipidomic profile from OA tissue was compared to other those obtained with RA and PsA samples: (a) OA vs PsA and (b) OA vs RA. OA synovial membranes were distinctively separated on the histogram score plot of PCA-DA from PsA and RA samples (Supplementary Fig. 6). The lipid entities with discriminatory potential were further evaluated for statistical significance. The lipid panel responsible of the discrimination between OA vs RA and PsA patients is summarized in Table II. Relative abundance values of those lipids are presented in Supplementary Table 3. Compared to RA and PsA, osteoarthritic synovial membranes were characterized by an increased abundance of PC. Specifically, PC (16:0_18:1) corresponding to $m / z 760.59$, and PC (18:1_18:1) related to $m / z$ 786.60 , were the highest discriminating markers. Additionally, OA tissues presented elevated levels of sphingomyelins such as SM (d18:1_16:0), SM (d18:1_24:1) and SM (d18:1_24:0), compared to RA (Fig. 5). On the other hand, among the lipids decreased in OA compared to PsA are PE-based plasmalogens, such as PE (P16:0_20:4), PE (P-18:1_20:4) and PE (P-18:0_22:6) [Fig. 6(A) and (B)]. Of the three plasmalogens identified to be altered between $\mathrm{OA}$ and PsA, two were increased in areas of the PsA synovial membranes characterized by inflammatory infiltrates [Fig. 6(C)]. Interestingly, two phosphatidic acids, PA (18:0_20:4) and PA (18:0_22:4), were also significantly decreased in OA compared to PsA.

\section{Discussion}

The composition and distribution of lipids in OA human synovial membranes have been analysed for the first time by MALDI-MSI.

The most remarkable characteristics of the OA tissue compared to healthy controls were found for PC. Specifically, PC (18:1_18:1) was significantly enriched in the hyperplastic synovial lining of OA. Similar PC species that we found altered in OA synovium were also increased in human OA FLS treated with growth factors such as TGF $\beta-1$ and IGF- $1^{23}$. Moreover, it has been reported that IL- $\beta 1$ markedly elevated the synthesis of certain PC species in human OA $\mathrm{FLS}^{24}$. The increased levels of PC observed in our study are in agreement with those findings and suggest an activation of the PC synthesis pathway in the LL of FLS within the OA synovium. Interestingly, an enhancement of the production of PC has also been recently associated to cardiac hypertrophy, suggesting a possible contribution of PC biosynthesis to the development of hypertrophic processes $^{25}$. Given that chondrocyte hypertrophy is known to be linked with the OA process ${ }^{26}$, further studies are warranted to elucidate the link of this phenotype with the increased amount of $\mathrm{PC}$ in synovium that has been unravelled in our work.

PC species are also increased in human OA SF compared with controls $^{12,27}$. Ten PC species that were found altered in early and late OA SF showed a significant higher abundance in the OA 
A

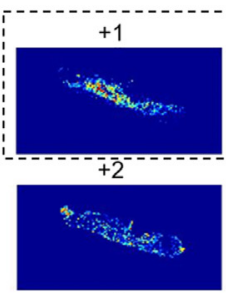

$+3$

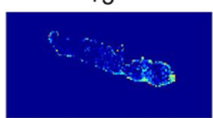

$+4$

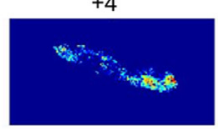

PC functions 1-4

C
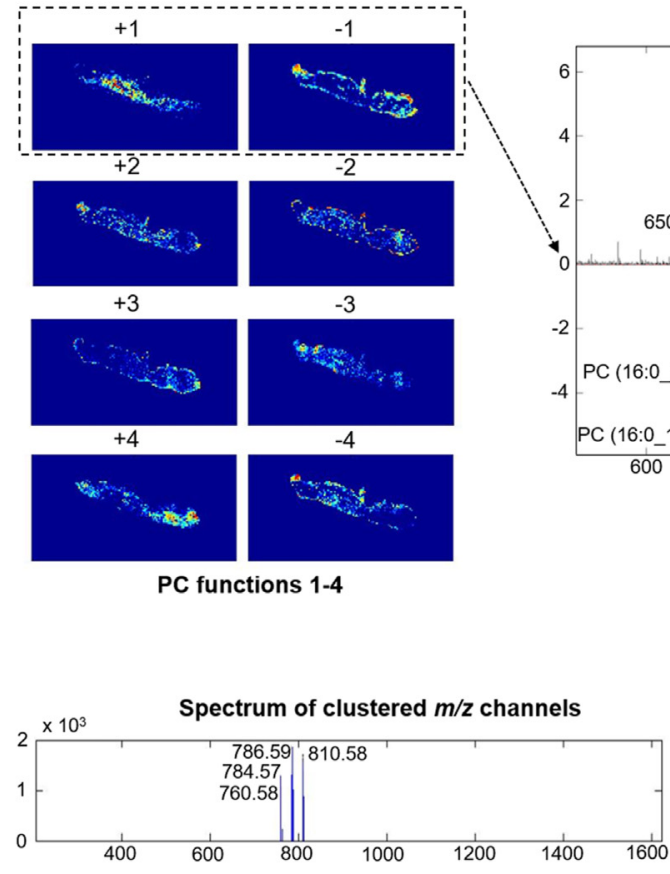

B

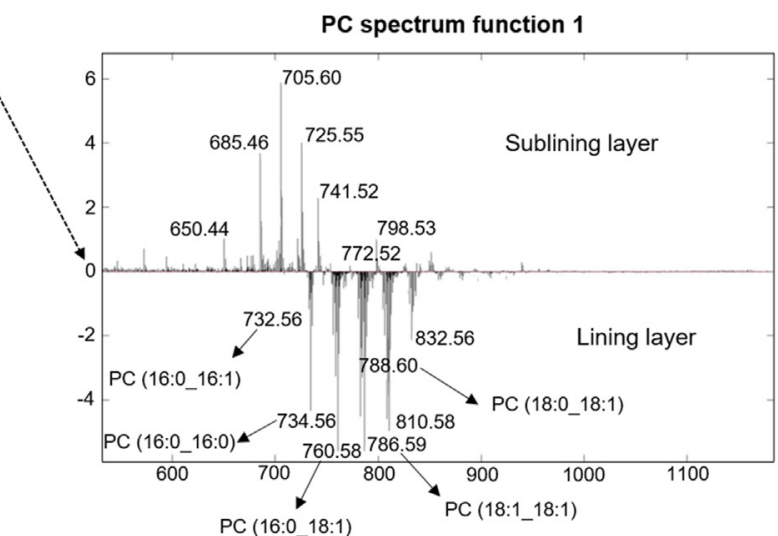

D

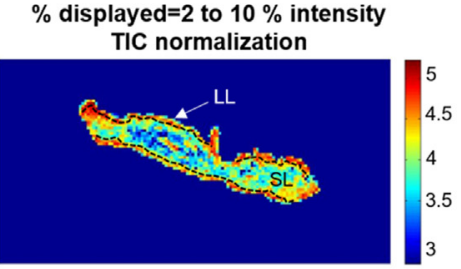

Correlation between the spatial distribution of phosphatidylcholines with the synovial lining layer in OA tissues. A) Principal component analysis (PCA) discriminated between OA synovial lining and sublining layers, according to PC lipid species. The figure shows principal component functions $1-4$ obtained by

Fig. 3 PCA. B) Principal component spectrum function one revealed that most of the $\mathrm{PC}$ are localized in the lining layer. C) Clustering analysis reported the co-localization of $\mathrm{m} / \mathrm{z} 760.59, \mathrm{~m} / \mathrm{z} 784.59, \mathrm{~m} / \mathrm{z} 786.60$ and $\mathrm{m} / \mathrm{z}$ 810.60, corresponding to PC lipid species, within OA tissues. D) Clustering image exhibited the higher intensity of PC in the synovial lining layer. LL: lining layer; SL: sublining layer; BV: blood vessels.

synovium of the present study ${ }^{12}$. Accordingly, another work demonstrated that levels of 24 GP (PC and LPC species) in SF were significantly higher in a subgroup of OA patients ${ }^{28}$. More recently, plasma levels of PC were significantly increased in a DMM model of OA and correlated with cartilage damage ${ }^{29}$. Similar to PC, LPC species were elevated in human OA SF ${ }^{12}$ and in an animal model of $\mathrm{OA}^{13}$. However, our data showed significantly lower amounts of LPC (16:0_0:0) and LPC (18:1_0:0) in the OA synovium compared to control tissue. This decreased abundance of LPC may appear controversial, given that the ratio of total LPC to PC in human plasma was previously reported as significantly increased in early ${ }^{30}$ and advanced ${ }^{31}$ OA patients. Nevertheless, the results obtained from those studies focused on exploring PC and LPC in OA are not consistent. In fact, a recent metabolomic analysis performed on serum showed no significant differences in the LPC to PC ratio between OA and controls ${ }^{32}$. Furthermore, LPC 18:2 and PC 44:3 have been recently identified as the specific lipid species whose ratio in serum is associated with the cartilage loss in $\mathrm{OA}^{33}$, none of which were identified in our untargeted analysis. This fact highlights the need of deeper studies on the structure of the different species of a given family of lipids, which is closely related with their biological function. It is expected that high-resolution MS technology would be extremely valuable to unravel the complex molecular configuration of lipids and their association with disease ${ }^{34}$.

In contrast with LPC, LPA 18:0 and LPA 18:1 appeared significantly increased in OA synovium compared to control. This indicates an upregulation of the LPA signalling, which has been extensively linked to inflammation and the promotion and maintenance of pain $^{35}$. In fact, there is evidence that LPA contributes to joint neuropathy and is involved in the development of neuropathic OA pain ${ }^{36}$. LPAs can be generated via the hydrolysis of the choline group from LPC by autotaxin (ATX) ${ }^{37}$. Interestingly, increased expression of both ATX and LPA has been reported in OA SF compared to controls and correlated with disease severity ${ }^{11,36,38}$. Thus, the production of LPA in the synovium and its accumulation in SF seems to contribute to OA pathogenesis and symptoms such as pain.

FA and FA-derived lipid mediators are proposed to play roles in the pathophysiology of joint diseases, contributing to articular inflammation $^{10,39}$. In fact, it has recently been described that the serum levels of polyunsaturated FA, including AA, positively correlate with markers of joint degeneration and systemic inflammation in $\mathrm{OA}^{40}$. Moreover, positive associations of serum AA levels with synovitis ${ }^{41}$ and histologic disease severity ${ }^{42}$ in knee OA have been reported. In our work, levels of AA, stearic acid and oleic acid were significantly increased in OA, suggesting an alteration of FA metabolism. In line with these results, other joint tissues (including the superficial area of the cartilage) have showed FA accumulation in $\mathrm{OA}^{43}$. Thus, the increment of FA in OA joint tissues constitutes a feasible source of pro-inflammatory cytokines and other lipid mediators that could promote synovitis.

Arthritic synovial tissues, including RA and PsA, typically have a more inflammatory phenotype with more infiltrating immune cells and a higher expression of cytokines than OA tissues ${ }^{44}$. Therefore, 


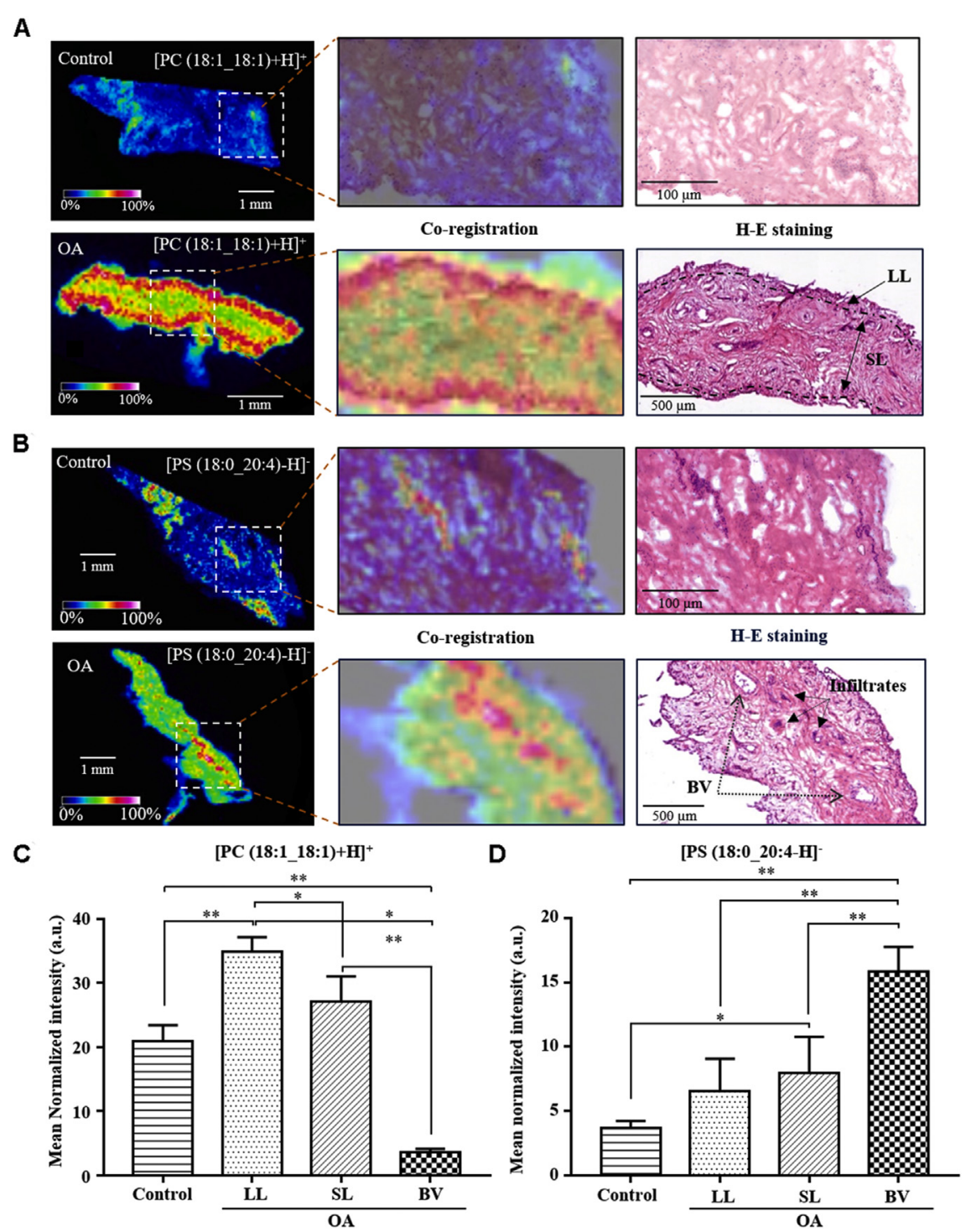

Phosphatidylcholine and phosphatidylserine distribution patterns in representative control and osteoarthritic synovial membranes. MALDI-MSI analysis showed specific glycerophospholipids with a preferential distribution to the lining layer (LL), sublining layer (SL) and blood vessels (BV). A) PC (18:1_18:1) $+\mathrm{H}^{+}$corresponding to $m / z 786.60$ is specifically localized in the $L L$. B) The spatial distribution of $\mathrm{m} / z 810.60$ assigned to PS (18:0_20:4) $-\mathrm{H}^{-}$seemed to be associated with areas of the OA sublining layer

Fig. 4 with increased vascularity and inflammatory infiltrates. $C$ and D) Quantification of variations in PC (18:1_18:1) $+\mathrm{H}^{+}$and PS (18:0_20:4)- $\mathrm{H}^{-}$intensities among the different synovial compartments in OA (LL: lining layer; SL, sublining layer; BV, blood vessels) compared to whole control samples. Values are the mean and Cartilage normalized intensity $\pm 95 \%$ confidence interval. * $P$-value $<0.05$; $* * P$-value $<0.01$. Bar length: $1 \mathrm{~mm}$. The colour scale at the bottom indicates the relative abundance. Pink or white denote higher abundances, while blue or black denote lower abundances. 


\begin{tabular}{|c|c|c|c|c|c|c|}
\hline $\mathrm{N}$ & & & OA vs RA & Significance & OA vs PsA & Significance \\
\hline & Sphingolipids & & & & & \\
\hline & Sphingomyelins (SM) & $m / z$ & & & & \\
\hline 1 & {$\left[S M\left(d 18: 1 \_16: 0\right)+H\right]^{+}$} & 703.58 & & $* *$ & & $*$ \\
\hline 2 & {$\left[\mathrm{SM}\left(\mathrm{d} 18: 1 \_16: 0\right)+\mathrm{Na}\right]^{+}$} & 725.56 & & $*$ & & 0.28 \\
\hline 3 & {$\left[S M\left(d 18: 1 \_16: 0\right)+K\right]^{+}$} & 741.53 & & $* *$ & & 0.43 \\
\hline 4 & {$\left[S M\left(d 18: 1 \_24: 1\right)+H\right]^{+}$} & 813.68 & & $* *$ & & 0.13 \\
\hline 5 & {$\left[S M\left(d 18: 1 \_24: 1\right)+\mathrm{Na}\right]^{+}$} & 835.67 & & $* *$ & & 0.25 \\
\hline \multirow[t]{3}{*}{6} & {$\left[\mathrm{SM}\left(\mathrm{d} 18: 1 \_24: 0\right)+\mathrm{Na}\right]^{+}$} & 837.68 & & $* *$ & & 0.82 \\
\hline & Glycerophospholipids & & & & & \\
\hline & $\begin{array}{l}\text { Lysophosphatidylcholines } \\
\text { (LPC) }\end{array}$ & & & & & \\
\hline 7 & {$\left[\mathrm{LPC}\left(16: 0 \_0: 0\right)+\mathrm{H}\right]^{+}$} & 496.34 & & 0.19 & & $*$ \\
\hline \multirow[t]{2}{*}{8} & {$\left[\mathrm{LPC}\left(18: 1 \_0: 0\right)+\mathrm{H}\right]^{+}$} & 522.36 & & $*$ & & $* *$ \\
\hline & Phosphatidic acids (PA) & & & & & \\
\hline 9 & [PA (18:0_20:4)-H] $^{-}$ & 723.50 & & 0.08 & & $* *$ \\
\hline \multirow[t]{2}{*}{10} & 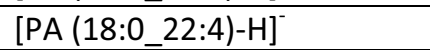 & 751.53 & & 0.68 & & $* *$ \\
\hline & Phosphatidylcholines (PC) & & & & & \\
\hline 11 & {$\left[\mathrm{PC}\left(16: 0 \_16: 1\right)+\mathrm{H}\right]^{+}$} & 732.55 & & $* *$ & & $*$ \\
\hline 12 & {$\left[\mathrm{PC}\left(16: 0 \_16: 1\right)+\mathrm{Na}\right]^{+}$} & 754.57 & & $* *$ & & 0.12 \\
\hline 13 & {$\left[\mathrm{PC}\left(16: 0 \_18: 2\right)+\mathrm{H}\right]^{+}$} & 758.57 & & * & & 0.90 \\
\hline 14 & {$\left[\mathrm{PC}\left(16: 0 \_18: 1\right)+\mathrm{H}\right]^{+}$} & 760.59 & & * & & $* *$ \\
\hline 15 & {$\left[\mathrm{PC}\left(16: 0 \_18: 0\right)+\mathrm{H}\right]^{+}$} & 762.60 & & $* *$ & & $*$ \\
\hline 16 & {$\left[\mathrm{PC}\left(0-18: 0 \_16: 1\right)+\mathrm{Na}\right]^{+}$} & 768.59 & & $* *$ & & $*$ \\
\hline 17 & {$\left[\mathrm{PC}\left(16: 0 \_16: 0\right)+\mathrm{K}\right]^{+}$} & 772.51 & & $* *$ & & 0.29 \\
\hline 18 & {$\left[\mathrm{PC}\left(16: 0 \_18: 2\right)+\mathrm{Na}\right]^{+}$} & 780.55 & & * & & 0.61 \\
\hline 19 & {$\left[\mathrm{PC}\left(18: 1 \_18: 2\right)+\mathrm{H}\right]^{+}$} & 784.59 & & $*$ & & 0.92 \\
\hline 20 & {$\left[\mathrm{PC}\left(18: 1 \_18: 1\right)+\mathrm{H}\right]^{+}$} & 786.60 & & $* *$ & & 0.32 \\
\hline 21 & {$\left[\mathrm{PC}\left(18: 0 \_18: 1\right)+\mathrm{H}\right]^{+}$} & 788.62 & & $* *$ & & $* *$ \\
\hline 22 & {$\left[\mathrm{PC}\left(18: 1 \_18: 2\right)+\mathrm{Na}\right]^{+}$} & 806.57 & & $*$ & & 0.67 \\
\hline \multirow[t]{2}{*}{23} & {$\left[\mathrm{PC}\left(18: 0 \_22: 6\right)+\mathrm{H}\right]^{+}$} & 834.60 & & $*$ & & 0.99 \\
\hline & $\begin{array}{l}\text { Phosphatidylethanolamines } \\
\text { (PE) }\end{array}$ & & & & & \\
\hline 24 & {$\left[\mathrm{PE}\left(\mathrm{P}-16: 0 \_20: 4\right)-\mathrm{H}\right]^{-}$} & 722.51 & & 0.86 & & $* *$ \\
\hline 25 & $\begin{array}{l}{\left[\mathrm{PE}\left(18: 0 \_18: 1\right)+\mathrm{H}\right]^{+} /[\mathrm{DMPE}} \\
\left.\left(16: 0 \_18: 1\right)+\mathrm{H}\right]^{+}\end{array}$ & 746.57 & & 0.38 & & $* *$ \\
\hline 26 & {$\left[\mathrm{PE}\left(\mathrm{P}-18: 1 \_20: 4\right)-\mathrm{H}\right]^{-}$} & 748.53 & & 0.64 & & $* *$ \\
\hline 27 & $\begin{array}{l}{\left[\mathrm{PE}\left(\mathrm{O}-16: 0 \_22: 4\right)-\mathrm{H}\right] /[\mathrm{PE}} \\
\left.\left(18: 0 \mathrm{p} \_20: 4\right)-\mathrm{H}\right]^{-}\end{array}$ & 750.54 & & 0.77 & & $* *$ \\
\hline \multirow[t]{2}{*}{28} & {$\left[\mathrm{PE}\left(\mathrm{P}-18: 0 \_22: 6\right)-\mathrm{H}\right]^{-}$} & 774.54 & & 0.26 & & $* *$ \\
\hline & Phophatidylinositols (PI) & & & & & \\
\hline 29 & {$\left[\mathrm{PI}\left(18: 0 \_22: 4\right)-\mathrm{H}\right]^{-}$} & 913.58 & & $*$ & & 0.36 \\
\hline
\end{tabular}

Lipid species were screened according to loading score values derived from PCA-DA analysis and tested (using Mann Whitney test) for statistical significance based on $p$-value $<0.05$. Red indicates increased lipid levels in OA compared to RA or PsA samples. Green indicates decreased lipid levels in OA compared to PsA samples. ${ }^{*} p$-value $<0.05 ;{ }^{* *} p$-value $<0.01$. 


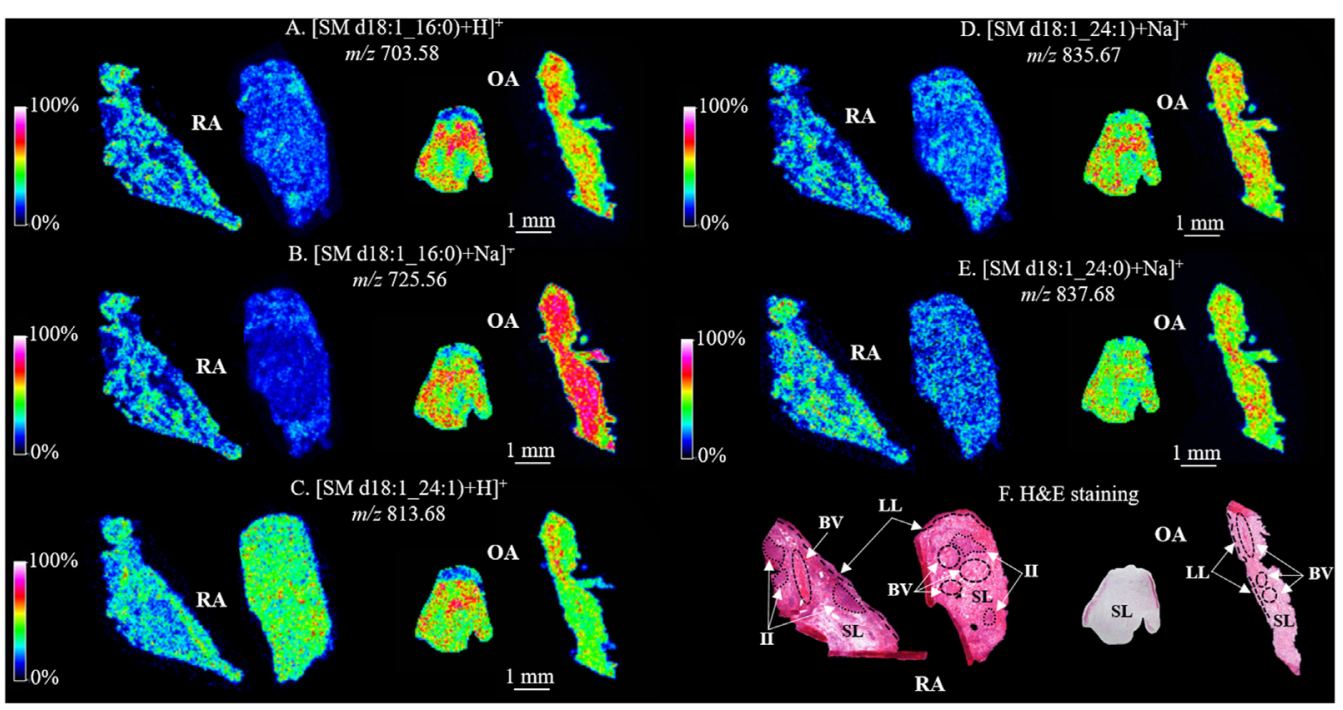

Increased SM abundance in OA synovial membranes related to RA tissues visualized by MALDIMSI. Images show samples from two representative RA and OA biposies. A-E) Relative intensity of selected SM species: SM d18:1_16:0, SM d18:1_24:1 and SM d18:1_24:0 shows higher levels in OA synovium. F) H\&E staining of RA and OA human synovial membranes. Bar length: $1 \mathrm{~mm}$. The colour scale on the left indicates the relative abundance. Pink or white denote higher abundances, while blue or black denote lower abundances. LL: lining layer; SL: sublining layer; BV: blood vessels; II: inflammatory infiltrates.

our initial hypothesis was that lipid levels would be higher in the synovium of patients with RA and PsA. Interestingly, this was not the case in our study for all lipid classes. The comparative analysis between these three pathological tissues allowed the identification of a lipidomic profile specific of OA and not general to inflammatory arthritis. Sphingolipids, including ceramide and sphingomyelin (SM) species, are nowadays recognized as relevant second messengers for signal-transduction pathways that regulate cell growth, differentiation, stress responses and programmed death in skeletal tissues ${ }^{45}$. Changes in SM plasma levels, specifically SM (d18:1_16:0), have been recently associated with cartilage damage in a mouse model of $\mathrm{OA}^{29}$, suggesting implications in joint diseases. Our study shows higher levels of SM species, including SM (d18:1_16:0), in OA compared with RA.

The present results also revealed PE-based plasmalogen species that are significantly raised in the synovial membrane of PsA patients compared to OA. Plasmalogens are a type of GP that can act as cell-protective antioxidants, preventing cell and lipid damage induced by oxidative stress ${ }^{46}$. Previous lipidomic studies showed that patients with RA and advanced OA have markedly increased levels of these species in the SF as compared to healthy individuals $^{12}$, but no data about PsA were available. Regarding their spatial distribution, several plasmalogens have been associated with both tumor and inflammation areas from colorectal cancer liver metastasis specimens using MALDI-MSI ${ }^{47}$. In our study, two PE-based plasmalogens also co-localize with heavily cell infiltratedrich areas of the PsA synovium. We hypothesize that the production of these lipids from the synoviocytes is increased in an attempt to reduce the inflammatory response in PsA.

This study opens opportunities for future tissue-based OA research but also has some limitations that need to be considered.
First, the high technological requirements of MSI do not allow performing large-scale studies, thus this analysis could be performed on a limited sample size $(n=41)$. This should be taken into account especially when interpreting the results, since it is unlikely that all lipidomic alterations could have been detected. Second, our study is limited by the lack of a true healthy control group, which might have precluded some findings. Nevertheless, as surgical intervention in the patients from the control group was performed months later after joint trauma, they did not present intra-articular inflammation at the time of arthroscopy. Third, we have not been able to record the nutritional behaviour or the drugs administered to the patients, which may have a potential impact on the lipid levels in their synovium. Therefore, further work is required to unequivocally distinguish disease-from putative diet or drugrelated lipid alterations. Finally, future studies should also correlate clinical markers of disease activity and radiographic changes with the synovium lipid profiles.

To conclude, this discovery study shows for the first time that OA synovial membranes can be discriminated from healthy tissues based on their lipidomic profiles, which have been characterized by MALDI-MSI. The major lipid disturbances in the OA synovium were elevated levels of certain GP, FA and LPA and lower levels of LPC. Furthermore, OA tissues were outlined by a higher content of PC and SM and a lower abundance of PE-based plasmalogens compared with other inflammatory joint diseases. Some of them co-localized with areas of high inflammation and vascularization, providing valuable information on their potential involvement in OA synovial pathophysiology. The alterations in the lipid signatures reported herein may contribute to the pathogenic processes that occur in OA and could support clinical decision-making for disease diagnosis and stratification, 


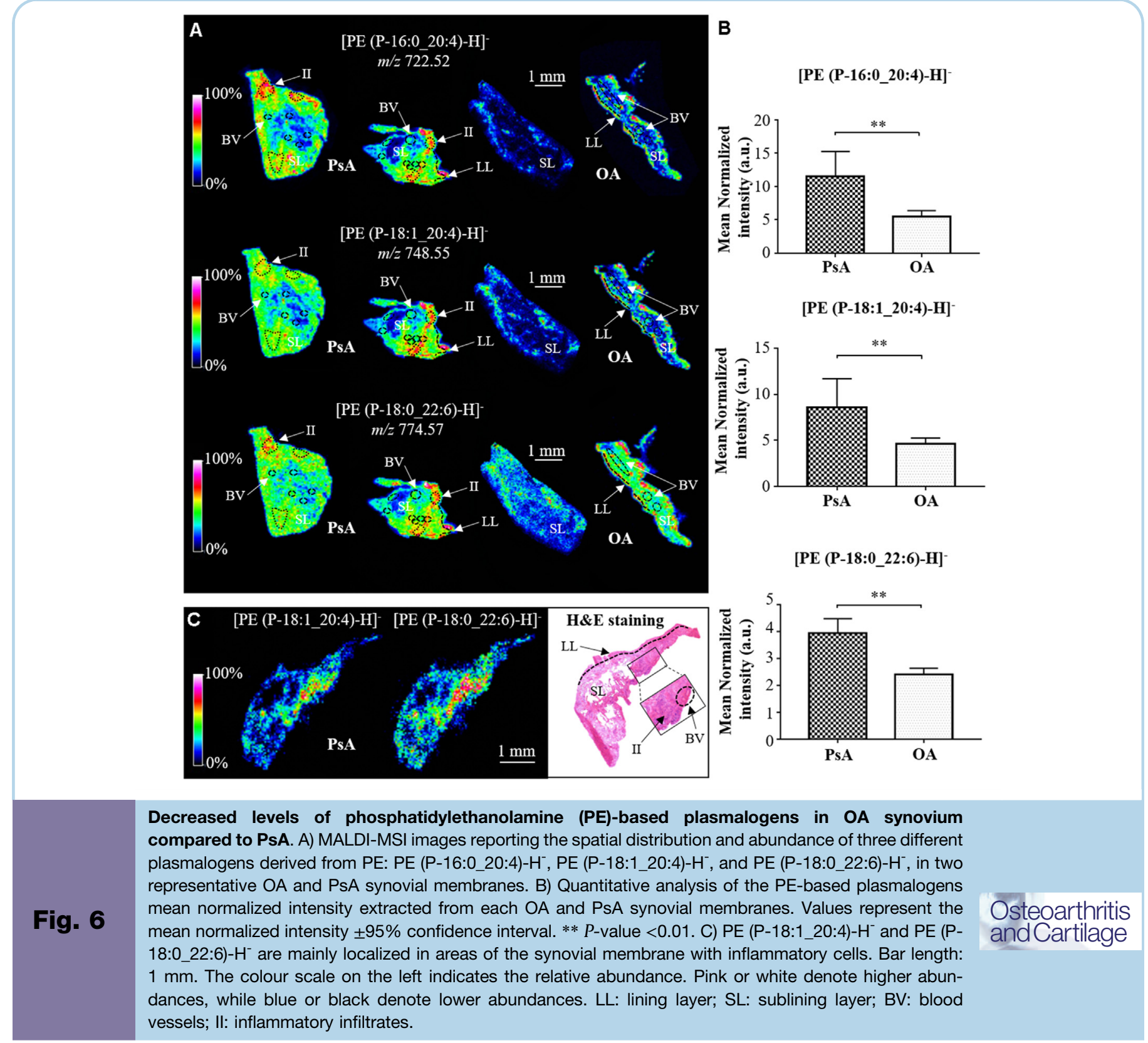

B

although they first need to be independently validated in further targeted studies.

\section{Data statement}

MALDI-MSI lipidomics data supporting the findings of this manuscript are available from the corresponding author upon request.

\section{Contributions}

All authors have made significant contributions to the conception and design of this study, the acquisition of data, its analysis and interpretation. All authors were involved in drafting the article and approved the final version to be published. Detailed contribution: study conception and design (B.R., C.R.R. and F.J.B.), data acquisition and analysis (B.R., B.C.P., R.M.A.H., M.R.L.P.), data interpretation and drafting of the manuscript (B.R., B.C.P., F.J.B. and C.R.R.).

\section{Competing interests}

We certify that there is no conflict of interest to disclose regarding the materials and data discussed in this manuscript. The contents of this manuscript have not been copyrighted or published previously.

\section{Role of funding source}

This work is supported by grants from Fondo Investigación Sanitaria-Spain (PI16/02124, PI17/00404 PI19/01206 and RETIC-RIERRD16/0012/0002), integrated in the National Plan for Scientific Program, Development and Technological Innovation 2013-2016 and funded by the ISCIII-General Subdirection of Assessment and Promotion of Research - European Regional Development Fund 
(FEDER) "A way of making Europe". This study is also supported by AE CICA-INIBIC (ED431E 2018/03) and grants IN607A2017/11 and IN607D2020/10 from Xunta de Galicia. The Biomedical Research Networking Center (CIBER) is an initiative from Instituto de Salud Carlos III (ISCIII). The Proteomics Unit of GIR belongs to ProteoRed, PRB3- ISCIII (PT17/0019/0014). B.R. has been supported by Xunta de Galicia (IN606B-2016/004). The research was partially performed within the M4I research program, financially supported by the Dutch Province of Limburg as part of the "LINK" program.

\section{Acknowledgements}

The authors express appreciation to the Histomorphology Service from INIBIC for providing the synovial membrane sections. We would also like to thank Vanesa Balboa Barreiro from the Research Support Unit of Complejo Hospitalario Universitario de A Coruña for providing assistance with data analysis.

\section{Supplementary data}

Supplementary data to this article can be found online at https://doi.org/10.1016/j.joca.2020.12.025.

\section{References}

1. Kraus VB, Blanco FJ, Englund M, Karsdal MA, Lohmander LS. Call for standardized definitions of osteoarthritis and risk stratification for clinical trials and clinical use. Osteoarthritis Cartilage 2015;23:1233-41.

2. Sellam J, Berenbaum F. The role of synovitis in pathophysiology and clinical symptoms of osteoarthritis. Nat Rev Rheumatol 2010;6:625-35.

3. Smith MD. The normal synovium. Open Rheumatol J 2011;5: 100-6.

4. Mathiessen A, Conaghan PG. Synovitis in osteoarthritis: current understanding with therapeutic implications. Arthritis Res Ther 2017;19:18.

5. Fernandes JC, Martel-Pelletier J, Pelletier JP. The role of cytokines in osteoarthritis pathophysiology. Biorheology 2002;39: 237-46.

6. Myers SL, Brandt KD, Ehlich JW, Braunstein EM, Shelbourne KD, Heck DA, et al. Synovial inflammation in patients with early osteoarthritis of the knee. J Rheumatol 1990;17:1662-9.

7. Ayral X, Pickering EH, Woodworth TG, Mackillop N, Dougados M. Synovitis: a potential predictive factor of structural progression of medial tibiofemoral knee osteoarthritis results of a 1 year longitudinal arthroscopic study in 422 patients. Osteoarthritis Cartilage 2005;13:361-7.

8. Wojdasiewicz P, Poniatowski Ł, Szukiewicz D. The role of inflammatory and anti-inflammatory cytokines in the pathogenesis of osteoarthritis. Mediat Inflamm 2014;2014:561459.

9. Lawrence T, Willoughby DA, Gilroy DW. Anti-inflammatory lipid mediators and insights into the resolution of inflammation. Nat Rev Immunol 2002;2:787-95.

10. Brouwers H, von Hegedus J, Toes R, Kloppenburg M, IoanFacsinay A. Lipid mediators of inflammation in rheumatoid arthritis and osteoarthritis. Best Pract Res Clin Rheumatol 2015;29:741-55.

11. Kosinska MK, Liebisch G, Lochnit G, Wilhelm J, Klein H, Kaesser U, et al. Sphingolipids in human synovial fluid-a lipidomic study. PloS One 2014;9, e91769.

12. Kosinska MK, Liebisch G, Lochnit G, Wilhelm J, Klein H, Kaesser U, et al. A lipidomic study of phospholipid classes and species in human synovial fluid. Arthritis Rheum 2013;65: 2323-33.

13. Kosinska MK, Mastbergen SC, Liebisch G, Wilhelm J, Dettmeyer RB, Ishaque B, et al. Comparative lipidomic analysis of synovial fluid in human and canine osteoarthritis. Osteoarthritis Cartilage 2016;24:1470-8.

14. Jurowski K, Kochan K, Walczak J, Barańska M, Piekoszewski W, Buszewski B. Analytical techniques in lipidomics: state of the art. Crit Rev Anal Chem 2017;47:418-37.

15. Rocha B, Ruiz-Romero C, Blanco FJ. Mass spectrometry imaging: a novel technology in rheumatology. Nat Rev Rheumatol 2017;13:52-63.

16. Schwamborn K, Caprioli RM. MALDI imaging mass spectrometry-painting molecular pictures. Mol Oncol 2010;4:529-38.

17. Walch A, Rauser S, Deininger SO, Höfler H. MALDI imaging mass spectrometry for direct tissue analysis: a new frontier for molecular histology. Histochem Cell Biol 2008;130: 421-34.

18. Orr C, Vieira-Sousa E, Boyle DL, Buch MH, Buckley CD, Cañete JD, et al. Synovial tissue research: a state-of-the-art review. Nat Rev Rheumatol 2017;13:463-75.

19. Altman R, Asch E, Bloch D, Bole G, Borenstein D, Brandt K, et al. Development of criteria for the classification and reporting of osteoarthritis. Classification of osteoarthritis of the knee. Diagnostic and Therapeutic Criteria Committee of the American Rheumatism Association. Arthritis Rheum 1986;29: 1039-49.

20. Aletaha D, Neogi T, Silman AJ, Funovits J, Felson DT, Bingham CO, et al. Rheumatoid arthritis classification criteria: an American College of Rheumatology/European League against Rheumatism collaborative initiative. Arthritis Rheum 2010;62:2569-81. 2010.

21. Taylor W, Gladman D, Helliwell P, Marchesoni A, Mease P, Mielants $\mathrm{H}$, et al. Classification criteria for psoriatic arthritis: development of new criteria from a large international study. Arthritis Rheum 2006;54:2665-73.

22. Eijkel GB, Kaletas B, Van der Wiel IM, Kros JM, Luinder TM, Heeren RMA. Correlating MALDI and SIMS imaging mass spectrometric datasets of biological tissue surfaces. Surf Interface 2009;41:675-85.

23. Sluzalska KD, Liebisch G, Wilhelm J, Ishaque B, Hackstein $H$ Schmitz G, et al. Growth factors regulate phospholipid biosynthesis in human fibroblast-like synoviocytes obtained from osteoarthritic knees. Sci Rep 2017;7:13469.

24. Sluzalska KD, Liebisch G, Lochnit G, Ishaque B, Hackstein $H$, Schmitz $G$, et al. Interleukin-1 $\beta$ affects the phospholipid biosynthesis of fibroblast-like synoviocytes from human osteoarthritic knee joints. Osteoarthritis Cartilage 2017;25: 1890-9.

25. Dong S, Zhang R, Liang Y, Shi J, Li J, Shang F, et al. Changes of myocardial lipidomics profiling in a rat model of diabetic cardiomyopathy using UPLC/Q-TOF/MS analysis. Diabetol Metab Syndrome 2017;9:56.

26. Dreier R. Hypertrophic differentiation of chondrocytes in osteoarthritis: the developmental aspect of degenerative joint disorders. Arthritis Res Ther 2010;12:216.

27. Kosinska MK, Ludwig TE, Liebisch G, Zhang R, Siebert HC, Wilhelm J, et al. Articular joint lubricants during osteoarthritis and rheumatoid arthritis display altered levels and molecular species. PloS One 2015;10, e0125192.

28. Zhang W, Likhodii S, Zhang Y, Aref-Eshghi E, Harper PE, Randell E, et al. Classification of osteoarthritis phenotypes by metabolomics analysis. BMJ Open 2014;4, e006286.

29. Pousinis P, Gowler PRW, Burston JJ, Ortori CA, Chapman V, Barrett DA. Lipidomic identification of plasma lipids associated 
with pain behaviour and pathology in a mouse model of osteoarthritis. Metabolomics 2020;16:32.

30. Castro-Perez JM, Kamphorst J, DeGroot J, Lafeber F, Goshawk J, Yu K, et al. Comprehensive LC-MS E lipidomic analysis using a shotgun approach and its application to biomarker detection and identification in osteoarthritis patients. J Proteome Res 2010;9:2377-89.

31. Zhang W, Sun G, Aitken D, Likhodii S, Liu M, Martin G, et al. Lysophosphatidylcholines to phosphatidylcholines ratio predicts advanced knee osteoarthritis. Rheumatology 2016;55: 1566-74.

32. Tootsi K, Vilba K, Märtson A, Kals J, Paapstel K, Zilmer M. Metabolomic signature of amino acids, biogenic amines and lipids in blood serum of patients with severe osteoarthritis. Metabolites 2020;10:323.

33. Zhai G, Pelletier JP, Liu M, Aitken D, Randell E, Rahman P, et al. Activation of the phosphatidylcholine to lysophosphatidylcholine pathway is associated with osteoarthritis knee cartilage volume loss over time. Sci Rep 2019;9:9648.

34. Cajka T, Smilowitz JT, Fiehn O. Validating quantitative untargeted lipidomics across nine liquid chromatography-highresolution mass spectrometry platforms. Anal Chem 2017;89: 12360-8.

35. Inoue M, Rashid MH, Fujita R, Contos JJ, Chun J, Ueda $H$. Initiation of neuropathic pain requires lysophosphatidic acid receptor signaling. Nat Med 2004;10:712-8.

36. McDougall JJ, Albacete S, Schuelert N, Mitchell PG, Lin C, Oskins JL, et al. Lysophosphatidic acid provides a missing link between osteoarthritis and joint neuropathic pain. Osteoarthritis Cartilage 2017;25:926-34.

37. Aoki J, Inoue A, Okudaira S. Two pathways for lysophosphatidic acid production. Biochim Biophys Acta 2008;1781:513-8.

38. Mabey T, Taleongpong P, Udomsinprasert W, Jirathanathornnukul N, Honsawek S. Plasma and synovial fluid autotaxin correlate with severity in knee osteoarthritis. Clin Chim Acta 2015;444:72-7.

39. Loef M, Schoones JW, Kloppenburg M, Ioan-Facsinay A. Fatty acids and osteoarthritis: different types, different effects. Joint Bone Spine 2019;86:451-8.

40. Wu CL, Kimmerling KA, Little D, Guilak F. Serum and synovial fluid lipidomic profiles predict obesity-associated osteoarthritis, synovitis, and wound repair. Sci Rep 2017;7:44315.

41. Baker KR, Matthan NR, Lichtenstein AH, Niu J, Guermazi A, Roemer $F$, et al. Association of plasma n-6 and n-3 polyunsaturated fatty acids with synovitis in the knee: the MOST study. Osteoarthritis Cartilage 2012;20:382-7.

42. Lippiello L, Walsh T, Fienhold M. The association of lipid abnormalities with tissue pathology in human osteoarthritic articular cartilage. Metabolism 1991;40:571-6.

43. Cillero-Pastor B, Eijkel G, Kiss A, Blanco FJ, Heeren RM. Timeof-flight secondary ion mass spectrometry-based molecular distribution distinguishing healthy and osteoarthritic human cartilage. Anal Chem 2012;84:8909-16.

44. de Lange-Brokaar BJ, Ioan-Facsinay A, van Osch GJ, Zuurmond AM, Schoones J, Toes RE, et al. Synovial inflammation, immune cells and their cytokines in osteoarthritis: a review. Osteoarthritis Cartilage 2012;20:1484-99.

45. Khavandgar Z, Murshed M. Sphingolipid metabolism and its role in the skeletal tissues. Cell Mol Life Sci 2015;72: 959-69.

46. Messias MCF, Mecatti GC, Priolli DG, de Oliveira Carvalho P. Plasmalogen lipids: functional mechanism and their involvement in gastrointestinal cancer. Lipids Health Dis 2018;17:41.

47. Patterson NH, Alabdulkarim B, Lazaris A, Thomas A, Marcinkiewicz MM, Gao ZH, et al. Assessment of pathological response to therapy using lipid mass spectrometry imaging. Sci Rep 2016;6:36814. 